\title{
On Integrating Device Level Schedules into Market Based Control
}

\author{
Jorrit S. Nutma*†, Gerwin Hoogsteen*, Albert Molderink*, \\ Wilco E. Wijbrandi ${ }^{\dagger}$, Johann L. Hurink*, Gerard J. M. Smit* \\ *Department of EEMCS, University of Twente, Enschede, the Netherlands \\ \{g.hoogsteen, a.molderink, j.l.hurink, g.j.m.smit\}@utwente.nl \\ ${ }^{\dagger}$ TNO, Groningen, the Netherlands \\ \{jorrit.nutma, wilco.wijbrandi\}@tno.nl
}

\begin{abstract}
To deal with challenges introduced by the adaption of distributed energy resources, Demand Side Management methodologies are being developed to ensure the availability and reliability of our electricity supply in the future. The PowerMatcher and TRIANA are two examples of such management methodologies. As the strengths of the approaches complement each other, the two approaches are combined in this research by developing a novel bidding strategy. This strategy is unique in the sense that it incorporates a device specific planning when the device bidding function is determined. Simulations show that the combination is capable of following a planning while performing real-time balancing to deal with prediction errors. It is shown that following a planning mitigates the effect of exploiting flexibility on undesired periods. In the use-case simulations, this results in a peak reduction of around $25 \%$.

Index Terms - demand side management, EV
\end{abstract}

\section{INTRODUCTION}

The adoption of Distributed Energy Resources (DER) by prosumers puts stress on distribution grids that were never designed to cope with large quantities of local energy generation. In addition, the envisioned electrification of heating and transportation will increase the stress on the electricity grid. As a reaction, concepts such as 'the Smart Grid' are introduced to increase the hosting capacity of existing grids for further integration of DER. Demand Side Management (DSM) is one of these smart grid technologies [1], which enables the control of domestic devices to influence the energy consumption/production. Within The Netherlands, two wellknown DSM methodologies are developed, namely TRIANA and the PowerMatcher. The former is a proactive methodology with predictions and planning capabilities on device level to optimize the energy profile for a longer time span. The PowerMatcher, on the other hand, is a real-time control approach, able to act fast on changing power profiles using an energy market with bidding functions. In this work, the combination of these two technologies is investigated. Although studies on this subject, such as [2] and [3], have been done before, the distinguishing approach of this paper is to integrate the device specific planning into the bidding function. This approach allows the system to reserve flexibility during real-time control to avoid problems in the near future.

The remainder of this paper is organized as follows. Section II covers the related work on DSM methodologies. A broad overview on how TRIANA and the PowerMatcher are combined is explained in Section III. Sections IV and V explain the theory behind the bidding functions and how local device schedules can be incorporated in the bidding strategy. To evaluate the performance of this system, Section VI gives details on the setup of a simulation experiment, the results of this experiment are presented in Section VII, and conclusions are drawn in Section VIII.

\section{RELATED WORK}

The interest in DSM methodologies is tremendous and has resulted in dozens of DSM methodologies, concepts and field tests with promising results as surveyed by Siano [1]. Molderink et al. [2] categorize DSM strategies based on two criteria. The first criterion is a distinction between online (realtime) and offline (planning) based control. The latter often requires a form of online control as device constraints may change over time, leading to infeasible plannings. The second criterion is the point of control: local or central. In central control approaches, various controllable devices are connected to a central entity that can send incentives to steer these devices. Usually, this is done in a distributed, tree-structured way to maintain a scalable system. In contrast, local control concerns a small subset of devices and uses only locally available information (e.g. within a single household).

A DSM approach is control by sending steering signals, such as prices, as stimuli to change the demand. Updated stimuli initiate a response from the loads and generators in the grid which optimize their momentary state. To realize such control, often a double-sided auction is used in which households communicate a bidding function that specifies the amount of energy they consume/produce for a certain signal/price. The controller, often called the auctioneer, aggregates these bids and selects a clearing price for which the market clears at the desired power consumption level. Such a double-sided auction approach is taken in the PowerMatcher [7]. This approach can be categorized as a central online approach.

Actions taken in the past may limit or exhaust the available flexibility in the future and, therefore, higher power peaks might arise. Proactive DSM methodologies, such as TRIANA [8], have been developed to circumvent these problems by using predictions and planning prior to the real events. This approach considers the amount of flexibility and therefore yields better optimization results [3]. 
A comparison study between two DSM approaches is executed by Claessen et al. [6]. Their results point out that using offline planning results in better load flattening performance compared to a pure online control strategy. Molderink et al. [2], [3] have studied the performance of different control strategies more in-depth. The authors compare a mathematical control approach (namely an Integer Linear Program) with an auction-based control approach as implementation of the real-time control step in TRIANA. The results point out that the auction-based approach combined with planning performs better in terms of achieving a flat overall power profile. Therefore, this research uses the same concept but takes a different approach in the sense that the device specific planning is incorporated in the bidding strategy.

The lack of predication and planning in the original PowerMatcher was the reason to develop a two-time-scale PowerMatcher [5]. In this approach, devices predict locally how much energy they expect to produce and consume in a given time period. The root controller aggregates this data to determine the average power consumption and the prices over the longer time scale. This information is sent back to the devices that incorporate the expected prices in their shorter time scale bidding function. The results show that the performance of the PowerMatcher is improved when it optimizes for cost minimization.

In [4], an auction based DSM approach is presented that can be categorized as a global online control strategy to solve the same problem. In this approach, constraints are predicted on a global level such that the law of large number applies, resulting in a good performance of the system.

\section{COMBININg TRIANA AND THE POWERMATCher}

\section{A. PowerMatcher}

The PowerMatcher [7] is an implementation of a scalable agent-based control methodology. The balancing mechanism of the PowerMatcher is based on microeconomics theory, i.e. the balancing is achieved by using a Walrasian auction of electric energy. Device agents send bidding functions in which they express for what price they want to consume how much power. Concentrators aggregate the bidding functions to create a scalable hierarchy and a system in which privacy is assured. Finally, all aggregated bids end up at the auctioneer which determines the Market Clearing Price (MCP). The MCP is essentially the steering signal that tells the devices what they should consume based on their bid. As the bids and market clearing only consider the given time interval, the methodology does not inherently take information about future events into account. This is the the major shortcoming of the PowerMatcher in comparison with TRIANA.

\section{B. TRIANA}

TRIANA is a three-step DSM methodology [8]. The three steps are: 1) prediction, 2) planning and 3) real-time control. The prediction step is used to investigate the potential of a group of households to attain a desired profile. The second step, planning, is used to schedule the predicted flexibility. These two steps are executed on a regular basis and use a

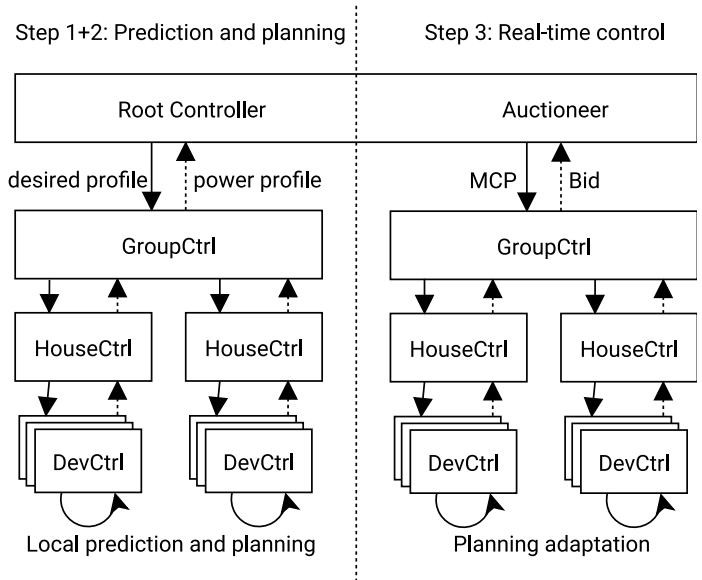

Fig. 1. Illustration of the control structure and signals in the three steps.

rolling horizon concept, resulting in a planned load profile for the upcoming day(s) or hour(s).

For the optimization of all flexibility, the profile steering approach as presented by Gerards et al. in [9] is used. Within a given planning horizon, the root controller steers the group of devices in an iterative fashion where privacy sensitive information is kept locally. To accomplish this, each individual device optimizes its behaviour based on the received steering signal with the use of local optimization algorithms, such as presented by van der Klauw et al. [10], and communicates this profile back. The control hierarchy matches the physical grid, ensuring that it operates within the grid capacity limits [11] and that it delivers a good power quality to end users [12]. The result of the planning phase is a schedule for each device, which can be aggregated to obtain an overall energy profile for a group of devices. However, these schedules are based on predicted constraints (e.g. charging deadline for an electric vehicle) and during run-time it can appear that these predicted constraints are not valid, resuling in infeasible schedules. Therefore, real-time control is required to resolve these infeasible schedules.

\section{Combining the two approaches}

In this work a method is proposed that combines the PowerMatcher and TRIANA by using the prediction and planning of TRIANA and providing it as input for the real-time control of the PowerMatcher methodology. Fig. 1 depicts the overall control structure and communication in the three steps.

The predictions are used as input for the planning phase which optimizes a group of devices towards a desired profile using the algorithm by Gerards et al. in [9]. The result of the planning phase is a local planning for each device for a given number of upcoming time intervals. Furthermore, the root controller in the tree structure aggregates all planned device profiles to an overall planning for the group of devices.

The third step, the online control, uses the concept of the PowerMatcher. Herein, the auctioneer is placed at the location of the root controller of the first two steps. The objective of the auctioneer is to follow the aggregated plan as good as possible. Each device has a planning which prescribes what it should 


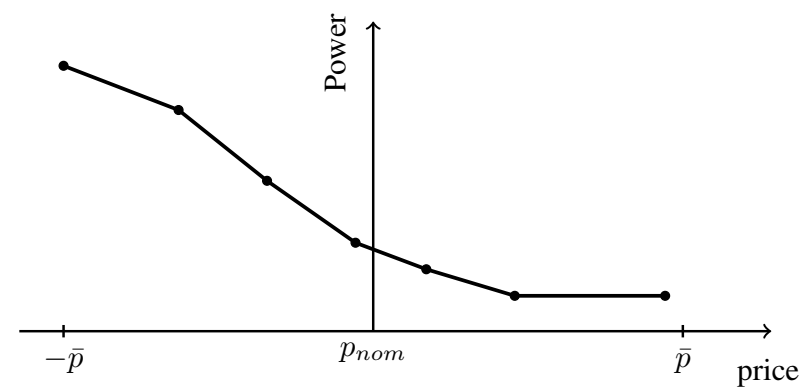

Fig. 2. Basic illustration of a bidding function in the combined DSM approach

consume/produce in the upcoming time intervals. Therefore, in the combined DSM approach, devices bid according to this plan, but also provide options to the auctioneer to cope with prediction errors. Devices will update the local planning based on run-time information.

\section{Fundamentals OF THE BIDDING FUNCTIONS}

Before presenting the novel bidding strategy that incorporates the device planning, a formal description of the bidding functions used is given:

Definition 1: A bidding function $b(p)$ is a monotonically decreasing function that gives for each price $p \in[-\bar{p}, \bar{p}]$ a power $P W \in \mathbb{R}$ :

$$
b(p): p \rightarrow P W
$$

Hereby, $\bar{p}>0$ is a given input.

The bidding function $b(p)$ is restricted in the sense that $b(p)$ gets characterized by the power values for a finite subset $\mathcal{P}=$ $\left\{p_{1}^{\prime}, . ., p_{N}^{\prime}\right\} \subset[-\bar{p}, \bar{p}]$, with $p_{i}^{\prime}<p_{i+1}^{\prime}$, for $i=1, . ., N-1$, $p_{1}^{\prime}=-\bar{p}$ and $p_{N}^{\prime}=\bar{p}$.

The basic approach is to use the given power values and interpolate between them. More precisely, for $p \in \mathcal{P}$, the value $b(p)$ is the given input value $\bar{b}(p)$ and for other prices $p$ the value $b(p)$ is calculated by interpolation between the two neighbouring points, i.e.:

$$
b(p)=b\left(p_{i}\right)+\left(p-p_{i}\right) \frac{b\left(p_{i+1}\right)-b\left(p_{i}\right)}{p_{i+1}-p_{i}} \text { if } p \in\left[p_{i}, p_{i+1}[.\right.
$$

Within the presented approach, bidding functions that are characterized as described above (Fig. 2 shows an example) are used. However, for devices that can only operate on a discrete number of power values, the above concept is adapted a bit, otherwise discontinuities occur. To prevent discontinuities, two restrictions are added. Firstly, $\mathcal{P}$ is restricted to integers. Secondly, devices with discontinuities round the received MCP to the nearest integer at the device level. This way, devices can explicitly specify for each power value the corresponding smallest and largest integer prices. As a result, a mix of continuous and discrete devices can be controlled using the same bidding method.

Bids of a group of devices or controllers can be aggregated in a hierarchical and scalable way as adding two valid bidding functions $b_{1}$ and $b_{2}$ results in a valid function $b_{3}=b_{1}+b_{2}$. Note that the value given by $b(p)$ is only valid for $p \in \mathbb{Z}$ for situations where discrete bidding functions are used.

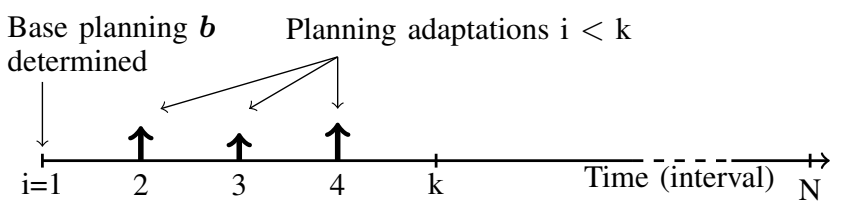

Fig. 3. Illustration to clarify the planning adaptation process at interval $\mathrm{i}=\mathrm{k}$

\section{A NOVEL BIDDING STRATEGY}

The result of a bidding strategy is a bidding function. In the proposed methodology, devices incorporate the device planning in the bidding strategy. The basic idea is that we normalize the bidding function around a nominal price $p_{n o m}$ (which we choose to be 0 ) and that each device positions its preferred power consumption at $p_{\text {nom }}$. Hereby, the preferred power consumption is the planned power value $P_{\text {plan }}$ of a device. Next to the planned power value, the bidding strategy provides other power options which are positioned at other prices. An example of such a bidding function is shown in Fig. 2.

\section{A. Two types of prediction errors}

The main objective of the taken real-time approach is to resolve prediction errors. Two types of prediction errors can be distinguished. The first type is referred to as event-based prediction errors, which are prediction errors that arise as the result of events in real-time. These can be resolved at run-time when information becomes available that provides sufficient information to determine the final planning of the device. As an example, when an Electric Vehicle (EV) arrives, the user submits the deadline and State-of-Charge (SoC) constraints of the $\mathrm{EV}$, and hence the planning can be updated according to the user input.

The second type of prediction errors are related to external phenomena which can occur continuously during the planning horizon, e.g. changing weather conditions that influence solar energy production. These errors are referred to as conditionbased prediction errors. In the end, the system has to cope both with event-based prediction errors and condition-based prediction errors.

\section{B. Planning adaptation}

As mentioned, devices put their planned power value at the nominal price but also offer options to deviate from the planning. In case that a device deviates from its planning, a compensation in the planning should be made, i.e. the planning should be adapted. In this way, the planning is updated and calculations for future planning adaptations can evaluate the costs of these deviations more accurately. In this section, the general form of planning adaptation is introduced and afterwards planning adaptation in case of event-based prediction errors and condition-based prediction errors is explained.

There are two domains related to the problem of planning adaptation, namely power and time. Some devices only offer flexibility in the time domain, e.g. smart dishwashers. Others in both the power and time domain, e.g. EVs. In this paper, 
we focus on power domain planning adaptation but the approach can easily be extended to allow time domain planning adaptation.

The basic idea for power domain planning adaptation is to deviate from the base planning as little as possible. A suitable criterion to evaluate a planning adaptation option for a device $\alpha$ is the squared euclidean distance with respect to the original planning. Assume that at time interval $k$, the following information is available:

- $\boldsymbol{b}=\left(b_{1}, b_{2}, \ldots, b_{N}\right)$, is the base planning of device $\alpha$, with $b_{i}$ being the planned power at interval $i$ and $N$ the number of time intervals in the planning horizon.

- $\boldsymbol{a}=\left(a_{1}, a_{2}, \ldots, a_{N}\right)$, is the adapted planning of device $\alpha$ at the beginning of time interval $k$ that is the result of options chosen in time intervals before $k$.

Furthermore, let $\boldsymbol{o}=\left(o_{1}, o_{2}, \ldots, o_{N}\right)$ be the power deviations of an adaptation option $\boldsymbol{o}$, which can be applied at time interval $k$, with respect to the planning $\boldsymbol{a}$ (see Fig. 3 for a time line with the events related to the planning adaptation till interval $k$ ). The base criterion for finding a suitable planning adaptation option $\boldsymbol{o}$ for device $\alpha$ at interval $k$ is given by:

$$
\min \operatorname{dev}_{\alpha, k}(\boldsymbol{o})=\sum_{i=k}^{N}\left(b_{i}-\left(a_{i}+o_{i}\right)\right)^{2} .
$$

1) Event-based planning adaptation: Event-based prediction errors are solved with a so-called event-based planning adaptation strategy. Note that for event-based planning adaptation, the adapted planning $\boldsymbol{a}$ is the same as the base planning $\boldsymbol{b}$ because up to that moment no auction-based planning adaptation has been applied, thus the optimization criterion boils down to:

$$
\min d e v_{\alpha, k}(\boldsymbol{o})=\sum_{i=k}^{N} o_{i}^{2} .
$$

Furthermore, in the case of an event-based prediction error, the device planning needs to be adapted such that the new planning complies to the current available information.

Mathematically, event-based planning adaptation is represented by a function $f$, which adapts the original planning, $\boldsymbol{b}$ of device $\alpha$. The result of the function is the event-based adapted planning $e$, which is saved at the device level next to the original planning.

$$
f(\boldsymbol{b})=e .
$$

An algorithm that solves (4), calculates the total difference in energy due to prediction errors and aims to spread this out over the available time till the deadline. The algorithm is schematically shown in Fig. 4.

2) Auction-based planning adaptation: In addition to eventbased prediction errors, the DSM approach should both cope with condition-based prediction errors and the effects of eventbased planning adaptation. The auctioneer does not adapt its planning, but, on the other hand, devices do adapt their local planning. Therefore, it is highly likely that the power at the nominal price in the aggregated bidding function does not

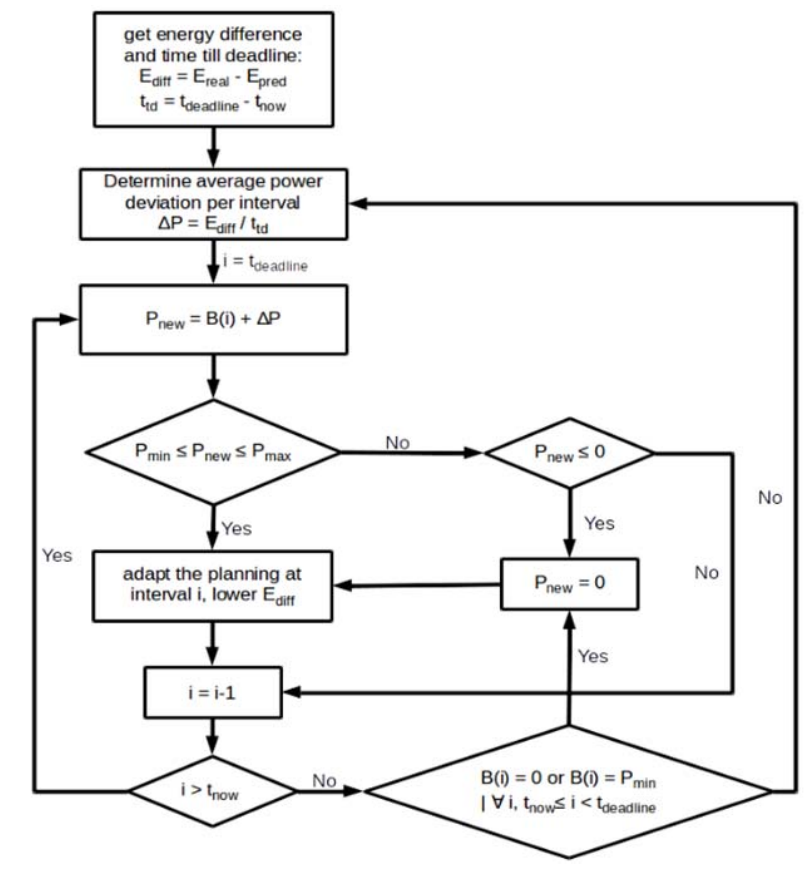

Fig. 4. Flowchart of an algorithm which spreads the deviations with respect to the planning out over all time intervals till the deadline.

match the planned power value at the auctioneer. Since the auctioneer wants to clear the market at the planned power value for the current time interval, each device should, if possible, also add extra options to the bidding function to provide flexibility.

So, in order to cope with prediction errors, devices are allowed to deviate from the planning. These deviation leads, again, to planning adaptations. The power options in the bidding function that correspond to planning adaptations should be positioned at prices that reflect how large the deviation from the planning for a particular option is. In this way, the auctioneer can distinguish between different planning adaptation options. To achieve this, a planning deviation option should be evaluated against the device planning. Therefore, each device does not only have the original planning, which is the result of TRIANA's planning phase, and an event-based adapted planning, but also an auction-based adapted planning.

In terms of the symbols occurring in (3), the event-based adapted planning is used as the base planning $(\boldsymbol{b})$ and a new, dynamically adapted auction-based planning is used as adapted planning $(\boldsymbol{a})$.

\section{Determining the bidding function}

A function $g$ is used to determine options for the planning adaption and additional options in the bidding function. This function takes an event-based adapted planning $e$ and an auction-based adapted planning $\boldsymbol{a}$ as input and provides a bidding function $b_{k}(p)$ at time interval $k$,

$$
b_{k}(p)=g(\boldsymbol{e}, \boldsymbol{a}) .
$$

The strategy to determine a bidding function $b_{k}(p)$ at time interval $k$, is explained in the remainder of this section. The 
basic idea of the strategy is to compensate for a power deviation in the current time interval. The strategy starts looking for compensation options at the deadline of the device and goes back to the current interval $k$. The reason to start at the end of the charging period is that compensating later in time results in extra time to cope with (other) prediction errors, which might undo the compensation. So the optimization criterion of (3) reduces to:

$$
\left.\min d e v_{\alpha, k}(j)=\left(e_{k}-\left(a_{k}+\Delta P\right)\right)^{2}+\left(e_{j}\right)-\left(a_{j}-\Delta P\right)\right)^{2},
$$

where $j$ is the time interval on which the compensation is positioned and $\Delta P$ is the difference between the planned power and the power of the planning adaptation option for time interval $k$. This strategy starts searching for the time interval of the deadline and goes iteratively back to time interval $k$. The time interval $j$ that yields the smallest $\operatorname{dev}(k)$ is the time interval in which the compensation will be placed.

The price corresponding to the chosen option is determined as follows: from (7), it follows that $\max ($ dev $)=2 * \Delta P_{\text {max }}^{2}$. With this maximum deviation, we scale dev of the chosen option to a price in the range: $p_{\text {dev }} \in\left[p_{\text {nom }}, . ., \bar{p}\right]$.

$$
p= \begin{cases}p_{\text {nom }}-\frac{\operatorname{dev} \cdot \beta \cdot \bar{p}}{\max (\operatorname{dev})} & \Delta P>0 \\ p_{\text {nom }}+\frac{\operatorname{dev} \cdot \beta \cdot \bar{p}}{\max (\operatorname{dev})} & \Delta P \leq 0\end{cases}
$$

Note that for $\beta>1$ the price obtained by (8) can be outside the price interval. In that case, the price should be set to the minimum or maximum value, depending on whether $\Delta P>0$ or not.

\section{Simulation Setup}

A simulation case is used to demonstrate the potential of the combined DSM approach. The input data for all the loads is provided using the Artificial Load Profile Generator (ALPG) as presented in [13]. This ALPG provides static load profiles and flexibility information such as arrival times and deadlines for EV charging and time-shiftable devices (e.g. washing machines). A futuristic case is considered, in which grid overloading is observed if no control is applied. Different amounts of smart white goods, EVs, PV panels and batteries are assigned to the households.

The simulation interval is 9 days, of which 7 days are used for analysis to leave out corner case effects of the simulation. The 7 days represent a winter week in the end of January/beginning of February. The interval length for the planning and online control is 15 minutes. A planning is made every 24 hours and spans 48 hours.

Three different real-time control approaches with peak shaving as objective are compared in this study:

- PowerMatcher only (PM): A simulation run in which the PowerMatcher without planning to clear the market at an average obtained power level of $39.95 \mathrm{~kW}$.

- PowerMatcher with global planning (PM-GP): A simulation with a global planning from TRIANA to clear the market at power values that are the result of predictions and planning optimizations. This approach is also taken in [2], [3].

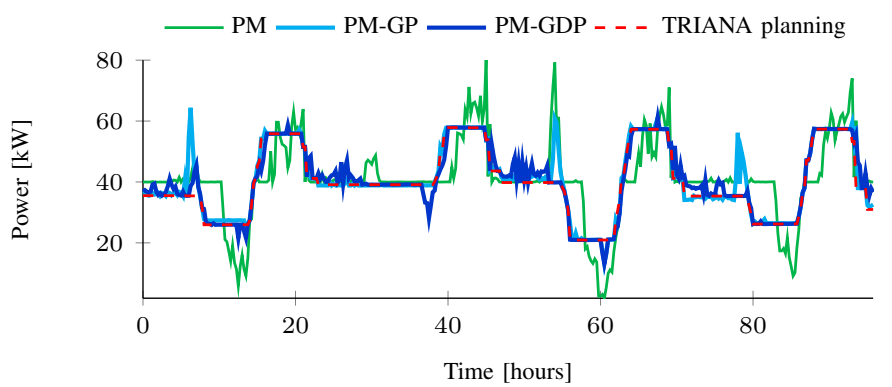

Fig. 5. Aggregated power profile of the three simulation cases.

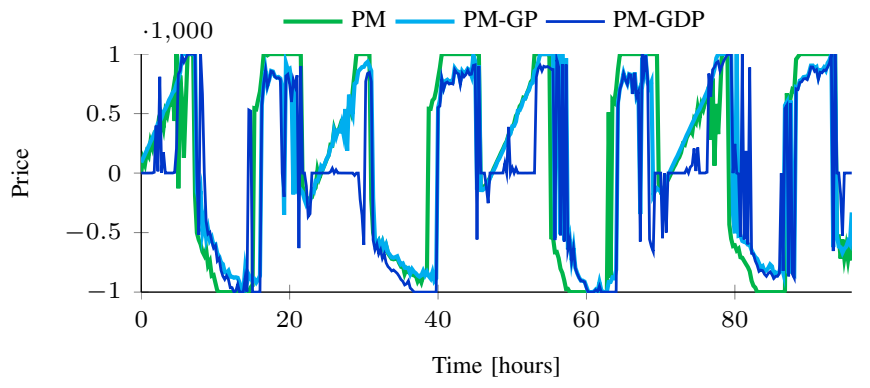

Fig. 6. MCP of the three simulations

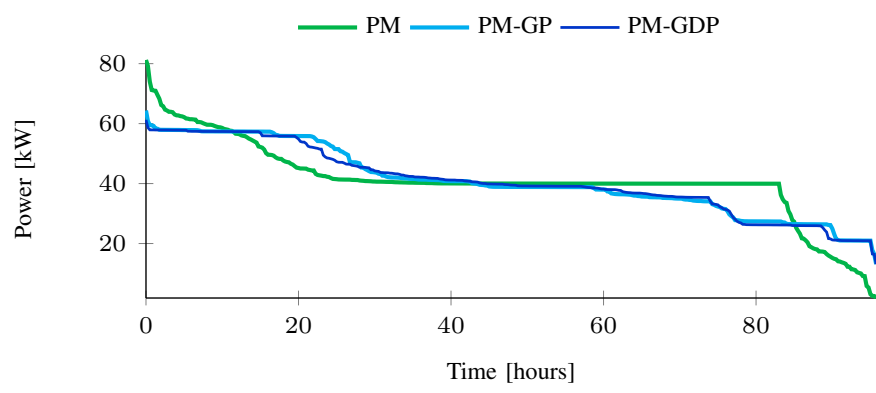

Fig. 7. Load duration curve the of aggregated power profile.

- PowerMatcher with global and device planning (PMGDP): In this simulation, the bidding strategy presented in this paper, which incorporates a device planning to determine the bidding function, is used.

\section{RESULTS AND DISCUSSION}

The results in Fig. 5 indicate that the PowerMatcher without planning results in the largest peak deviations. The peaks of PM-GP occur during the morning peaks. This results from the fact that the batteries are discharged too early with respect to the planning and, consequently, the buffers do not contain enough energy to lower the morning peak. This effect is caused by part of the appliances being predicted not to run at all, which means that too little energy in the planning horizon is predicted.

This mismatch also results in the extreme MCP for large parts of the simulation (Fig. 6). Time-shiftable devices start to defer their run-times because of the high MCP, until their latest possible starting time. Since this time is rougly the same 
TABLE I

QUANTITATIVE SIMULATION RESULTS. 7 DAYS OF SIMULATION.

\begin{tabular}{l||l|l|l} 
& PM-GDP & PM-GP & PM \\
\hline \hline Off the plan: & $3.47 \mathrm{E}+09$ & $3.44 \mathrm{E}+09$ & \\
Off the average: & $8.79 \mathrm{E}+10$ & $8.90 \mathrm{E}+10$ & $4.75 \mathrm{E}+10$ \\
Energy consumed $(\mathbf{M W h})$ & 6.66 & 6.69 & 6.96 \\
Peak deviation from avg $(\mathbf{k W})$ & 31.9 & 28.9 & 42.4 \\
Valley deviation from avg (kW) & 26.5 & 26.7 & 40.3 \\
Max peak power $(\mathbf{k W})$ & 71.6 & 78.7 & 83.8 \\
Min valley power $(\mathbf{k W})$ & 13.1 & 13.1 & 1.2
\end{tabular}

for most devices, a synchronized load peak occurs.

Above mentioned observations are also clearly visible in the load duration curve depicted in Fig. 7. The results of PM-GDP show the smallest peaks as the deviations are more spread out, which resolves the peaks seen with PM-GP. It has to be noted that the difference between PM-GDP and PM-GP is small.

Numerical simulation results are given in table I. In this table, the values for 'off the plan' and 'off the average' are determined as follows. For every interval, the difference between the planned power value and real power consumption is squared and summed to get one number for the whole simulation run. The values 'peak deviation from average' are the difference between the maximum occurring aggregated power value and the average power, so the maximum positive difference. Similarly, the 'valley deviation from average' gives the minimum negative difference between aggregated power and the aggregated average power.

As shown in Table I, the peak deviation is reduced with $25 \%$ when the PM-GDP strategy is used compared to the PowerMatcher simulation. Furhtermore, the maximum peak values of the the PowerMatcher are reduced with $15 \%$ in the PM-GDP strategy. Note that the peak of PM-GDP occurred in an interval that is not shown in Fig. 5 because the figure only shows four days out of seven days considered. The squared euclidean distance between the average and the power curve is smaller for the PowerMatcher than for the other approaches.

The aggregated power profile of PM-GDP is improved with respect to the one of PM-GP, but the remark should be made that it comes at higher costs in terms of implementation work. A consequence of the PM-GDP approach is that the MCP cannot be interpreted as a mere economic steering price. This is disadvantageous because it makes it more difficult to integrate the approach within electricity markets. On the other hand, the MCP in the PM-GDP approach gives new information about the system state because it reflects how far the devices diverge form the planning. This information can be used by global controllers to adapt their planning or by device controllers to incorporate this information in the bidding strategy. Finally, a completely other approach to cope with prediction errors is to not adapt any local planning but to perform a replanning more often. That approach could make use of exiting planning algorithms but requires a way to deal with the trade-off between, on the one hand, doing a replanning for each and every prediction error and, on the other hand, allowing a device to deviate from the planning if prediction errors occur.

\section{CONCLUSION}

The combination of the DSM methodologies the PowerMatcher and TRIANA leads to a DSM approach that is more comprehensive than the individual methodologies themselves. The planning from TRIANA, which is based on predictions, can be provided to a PowerMatcher implementation, resulting in an approach that follows the planning while dealing with prediction errors. This is an improvement with respect to the PowerMatcher because it provides a powerful way to achieve not only a momentary balancing of energy, which is a strength of the PowerMatcher, but also a solution that is optimized taking a larger time span into account. The simulation results point out that this indeed results in a flatter profile as flexibility is used better, i.e. the effect of exploiting flexibility on moments that lead to balancing problems later on is mitigated. The simulation results of the comparison study show that the largest peak deviation from the average is reduced by $25 \%$ if the combined approach is used, compared to the PowerMatcher only approach.

It is left for future work to implement planning adaptation strategies for time-shiftable devices and to use the information expressed by the MCP to adapt the global planning as well.

\section{REFERENCES}

[1] P. Siano, "Demand response and smart grids a survey", Renewable and Sustainable Energy Reviews, vol. 30, pp. 461-478, 2014.

[2] A. Molderink, V. Bakker, J. L. Hurink and G. J. M. Smit, "On indirect controlled cost function based DSM strategies," PowerTech (POWERTECH), 2013 IEEE Grenoble, Grenoble, 2013, pp. 1-6.

[3] A. Molderink, V. Bakker, J.L. Hurink and G.J.M. Smit, "Comparing demand side management approaches," Innovative Smart Grid Technologies (ISGT Europe), 2012 3rd IEEE PES International Conference and Exhibition on , vol., no., pp.1,8, 14-17 Oct. 2012

[4] F. Ruelens et al., "Demand side management of electric vehicles with uncertainty on arrival and departure times," 2012 3rd IEEE PES Innovative Smart Grid Technologies Europe (ISGT Europe), Berlin, 2012, pp. 1-8.

[5] Y. Prins, "Dynamic programming in the PowerMatcher", MSc thesis. University of Twente and TNO, 2015.

[6] F.N. Claessen, B. Claessens, M.P.F. Hommelberg, A. Molderink, V. Bakker, H.A. Toersche, M.A. van den Broek, Comparative analysis of tertiary control systems for smart grids using the Flex Street model, Renewable Energy, Volume 69, Sept. 2014, Pages 260-270, ISSN 09601481

[7] K. Kok, "the PowerMatcher: Smart Coordination for the Smart Electricity Grid", PhD dissertation, Vrije Unviversiteit Amsterdam, 2013

[8] A. Molderink, V. Bakker, M.G.C. Bosman, J.L. Hurink and G.J.M. Smit, "Management and control of domestic smart grid technology," Smart Grid, IEEE Transactions on, vol.1, no.2, pp.109-119, Sept. 2010

[9] M.E.T. Gerards, H.A. Toersche, G. Hoogsteen, T. van der Klauw, J.L. Hurink and G.J.M. Smit, "Demand side management using profile steering", PowerTech (POWERTECH), 2015 IEEE Eindhoven, 29 Jun-2 Jul. 2015

[10] T. van der Klauw, M. E. T. Gerards, G. J. M. Smit and J. L. Hurink, "Optimal scheduling of electrical vehicle charging under two types of steering signals," IEEE PES Innovative Smart Grid Technologies, Europe, Istanbul, 2014, pp. 1-6.

[11] T. van der Klauw, M.E.T. Gerards, G. Hoogsteen, G.J.M. Smit and J.L. Hurink, "Considering grid limitations in profile steering," 2016 IEEE International Energy Conference (ENERGYCON), Leuven, 2016, pp. 1-6.

[12] G. Hoogsteen, A. Molderink, J. L. Hurink and G.J. M. Smit, "Managing energy in time and space in smart grids using TRIANA," IEEE PES Innovative Smart Grid Technologies, Europe, Istanbul, 2014, pp. 1-6.

[13] G. Hoogsteen, A. Molderink, J.L. Hurink and G.J.M. Smit, "Generation of Flexible Domestic Load Profiles to Evaluate Demand Side Management Approaches," in EnergyCon, 2016 IEEE Leuven, 4-8 Apr. 2016 